\title{
Detection of Extended Spectrum Betalactamases (ESBLs) Producing Enterobacteriaceae from Clinical Samples of Pus
}

\author{
K.R. Shobha Medegar, Y.K. Harshika* and Asha B. Patil \\ Department of Microbiology Karnataka Institute of Medical Sciences, \\ Vidyanagar, Hubli - 580022, India
}

*Corresponding author

\begin{abstract}
A B S T R A C T
Keywords

Enterobacteriaceae, Drug resistance, ESBL, DDST, Pus

Article Info

Accepted:

10 December 2018

Available Online:

10 January 2019

Extended spectrum $\beta$-lactamase (ESBL) producing Enterobacteriaceae has tremendously increased worldwide and it is one of the most common causes of morbidity and mortality associated with hospital-acquired infections. This could be attributed to association of multi drug resistance in ESBL producing isolates. Therefore, in the present study, an attempt was made to know the rate of ESBL producing Enterobacteriaceae and to know their antibiogram at Dr. B.R. Ambedkar Medical College. Bangalore. All the patients with signs and symptoms suggestive of abscess, wound infection, otitis media were included in the study. Pus samples from these patients were processed with standard microbiological procedures and the isolates were screened for ESBL production by Double disk synergy method. Out of 100 isolates, 19 isolates were ESBL producers. The most common ESBL producers were E. coli followed by Klebsiella, Citrobacter and Enterobacter. Majority of ESBL producers showed resistance to Ampicillin, Amoxyclav, cotrimoxazole and Amikacin. The production of ESBLs by clinically important isolates is emerging as a wide spread problem in our set up. Hence, routine detection, appropriate infection control measures are needed to stem the spread of this emerging form of resistance.
\end{abstract}

\section{Introduction}

Resistant bacteria are emerging worldwide as a threat to the favourable outcome of common infections in community and hospital settings. $\beta$-lactamase production by several gram negative and gram positive organisms is perhaps the most important single mechanism of resistance to penicillins and cephalosporins. ${ }^{1}$

There has been increased incidence and prevalence of extended spectrum $\beta$-lactamases (ESBLs), enzymes that hydrolyse and cause resistance to oxyiminocephalosporins and aztreonam $^{3}$. They represent a major group of $\beta$-lactamases belonging to Ambler class A penicillinases currently being identified worldwide in large numbers and now found in a significant percentage of Escherichia coli and Klebsiella pneumoniae strains. They have also been found in other Enterobacteriaceae strains like Enterobacter, Citrobacter, Proteus, Morgenella morganii, Serratia marsescens, Shigella dysenteriae, Pseudomonas aeruginosa. ${ }^{3}$ Today over 150 different ESBLs have been described. ${ }^{2}$ 
Major risk factors for colonization or infection with ESBL producing organism are long term antibiotic exposure, prolonged hospital stay, severe illness, and resistance in an institution with high rates of third generation cephalosporin use and instrumentation or catheterization. $^{1}$

It is necessary to know the prevalence of these strains in a hospital so as to formulate a policy of empirical therapy in high risk units. Equally important is to procure information on an isolate from a patient to avoid misuse of extended spectrum cephalosporins.

\section{Materials and Methods}

\section{Source of data}

All patients admitting and/or attending the outpatient department in Dr.B.R. Ambedkar Medical College Hospital with abscess, wound infection, otitis media, were the source of study.

Pus collected from such affected sites constituted the material of study.

Detailed history and clinical findings were recorded in the proforma.

\section{Inclusion criteria}

All age groups and both sexes having suspected pyogenic infections were included in the present study.

Only those cases yielding growth of Enterobacteriaceae from the cultured pus was included in the study and was further tested for ESBL production.

\section{Exclusion criteria}

Cases of pyogenic infections which did not yield the growth of Enterobacteriaceae, but yielded growth of other bacteria were not included.

\section{Sample processing}

The samples collected were processed in our laboratory using Standard microbiological procedures. The samples were subjected for Gram Stain and Culture. The culture media used were Chocolate Agar, Mac Conkey Agar and Thioglycollate Medium (Hi-Media, Mumbai India) to obtain isolated colonies.

\section{Isolation of enterobacteriaceae}

Isolates were identified based on colony morphology, motility, relevant biochemical reactions such as Catalase test, Oxidase test, Sugar Fermentation test (glucose, sucrose, maltose, lactose), Hugh Leifsons oxidation fermentation test, nitrate reduction test, indole production, methyl red test, Voges Proskauer test, citrate utilization test, urease test, triple sugar iron agar test, phenylalanine deaminase test, aminoacid decaroxylation test.

Following gram negative organisms isolated from clinical specimens were included in the study: Escherichia coli, Klebsiella, Citrobacter, Enterobacter, Proteus, Serratia and Morganella species.

\section{Antibiotic susceptibility testing}

Enterobacteriaceae isolates were subjected for antibiotic susceptibility testing by Kirby Bauer disk diffusion technique. In the present study susceptibility was tested against Ampicillin $(10 \mu \mathrm{g})$, Amikacin $(30 \mu \mathrm{g})$, Amoxyclav (30 $\mu \mathrm{g})$, Cotrimoxazole $(1.25 / 23.75 \mu \mathrm{g})$, Imipenem $(10 \mu \mathrm{g})$, Ciprofloxacin $(5 \mu \mathrm{g})$, Netilmicin $(10 \mu \mathrm{g})$, Ceftazidime $(30 \mu \mathrm{g})$, Cefotaxime $(30 \mu \mathrm{g})$, Cefpodoxime $(30 \mu \mathrm{g})$. These discs were obtained from HiMedia laboratories Pvt. Ltd - Mumbai. 
The diameter of zone of inhibition was measured and interpreted according to the guidelines of NCCLS guide lines.

\section{Double Disc Diffusion Synergy Test (DDST)}

All isolates belonging to Enterobacteriaceae were tested for ESBL production by DDST. Ceftazidime $(30 \mu \mathrm{g})$, Cefotaxime $(30 \mu \mathrm{g})$, Cefpodoxime $(30 \mu \mathrm{g})$ and Co-Amoxyclav (Amoxicillin $20 \mu \mathrm{g}+$ Clavulanic acid $10 \mu \mathrm{g}$ ) (HiMedia Laboratories Ltd. Mumbai) were used for ESBL detection.

Klebsiella pneumoniae ATCC 700603 and Escherichia coli ATCC 25922 were used as positive and negative controls respectively.

The suspension for inoculation was prepared from 4-5 isolated colonies and turbidity was compared with 0.5 McFarland standards. Sterile cotton swab soaked in this suspension was used to make lawn culture on Mueller Hinton agar plates. Co-Amoxyclav (20 $\mu \mathrm{g}$ Amoxicillin $+10 \mu \mathrm{g}$ Clavulanic acid) and Ceftazidime $(30 \mu \mathrm{g})$ were placed at distance of $20 \mathrm{~mm}$ from centre to centre. Plates were incubated at $37^{\circ} \mathrm{C}$ overnight. Enhancement of zone of inhibition of the ceftazidime towards the Co-Amoxyclav disc was considered positive result. This occurs because the clavulanic acid present in Co-Amoxyclav disc inactivates ESBL produced by the test organism.

\section{Results and Discussion}

The present study was carried out in the department of Microbiology, Dr.B. R. Ambedkar Medical College, Bangalore, between November 2009 and November 2010 to look for the presence of ESBL (Extended spectrum beta lactamases) in Enterobacteriaceae isolated from pus samples. A total of 100 isolates of Enterobacteriaceae were studied.
Out of 100 isolates, commonest isolates were E. coli (42\%), Klebsiella (27\%), and Proteus (11\%). Others like Enterobacter, Citrobacter, Serratia and Morgenella species were less commonly isolated.

In the females, the maximum number of cases were isolated between the age group of 21$30 y r s$ (26\%) followed by 51-60yrs (23\%). In the males, maximum numbers of cases were isolated between the age group of 31-40yrs (23\%) and 51-60yrs (23\%). Out of 100 cases, $57 \%$ (57) were males and 43\% (43) were females. The male to female ratio was $1.32: 1$ (Fig. 1 and Table 1).

Maximum number of isolates (24\%) were isolated from cases of abscess, followed by $18 \%$ from cases of postoperative infection.10$11 \%$ of the isolates were isolated from CSOM, diabetic ulcer and nonhealing ulcer. Around 1$6 \%$ isolates were isolated from burns, cellulitis, gangrene, osteomyelitis and sinusitis.

Among 100 cases of Enterobacteriaceae, 19 (19\%) were ESBL producers. In the present study E. coli (24\%) was common ESBL producer. Other ESBL producers were Klebsiella (22\%), Citrobacter (17\%), Enterobacter (11\%), and Proteus species (9\%). No ESBL was isolated from Serratia and Morganella species.

$30-50 \%$ of ESBL producers were isolated from gangrene, cellulitis, nonhealing ulcer. $20 \%$ were isolated from Diabetic ulcer and osteomyelitis. $17-22 \%$ of ESBL producers were isolated from abscesses and postoperative infection. No ESBL isolates were identified from burns, CSOM and sinusitis.

In the present study, all the 19(19\%) ESBL producers showed $100 \%$ resistance to A (Ampicillin), Ac (Amoxyclav). All the isolates were sensitive to Imipenem. They showed 
variable resistance pattern to Tobramycin (89.47\%), Cotrimoxazole Cefotaxime
(78.95\%),

Cefpodoxime
(78.95\%), Ceftazidime (73.68\%), Amikacin (68.42\%), Gatifloxacin (63.16\%) (Fig. 2 and Graph 1-4).

Table.1 Age and sex distribution of Enterobacteriaceae

\begin{tabular}{|c|c|c|c|c|c|c|}
\hline \multirow{2}{*}{$\begin{array}{l}\text { Age in } \\
\text { years }\end{array}$} & \multicolumn{4}{|c|}{ Sex } & \multirow[t]{2}{*}{ Total } & \multirow{2}{*}{$\begin{array}{l}\text { Total } \\
\text { Percentage }\end{array}$} \\
\hline & $\mathrm{F}$ & Percentage & M & Percentage & & \\
\hline $1-10$ & 4 & 9 & 4 & 7 & 8 & 8 \\
\hline $11-20$ & 1 & 2 & 0 & 0 & 1 & 1 \\
\hline $21-30$ & 11 & 26 & 9 & 16 & 20 & 20 \\
\hline $31-40$ & 7 & 16 & 13 & 23 & 20 & 20 \\
\hline $41-50$ & 4 & 9 & 11 & 19 & 15 & 15 \\
\hline $51-60$ & 10 & 23 & 13 & 23 & 23 & 23 \\
\hline $61-70$ & 5 & 12 & 5 & 9 & 10 & 10 \\
\hline $71-80$ & 0 & 0 & 2 & 4 & 2 & 2 \\
\hline $81-90$ & 1 & 2 & 0 & 0 & 1 & 1 \\
\hline Total & 43 & 100 & 57 & 100 & 100 & 100 \\
\hline
\end{tabular}

Fig.1 Antibiogram of ESBL producer strain

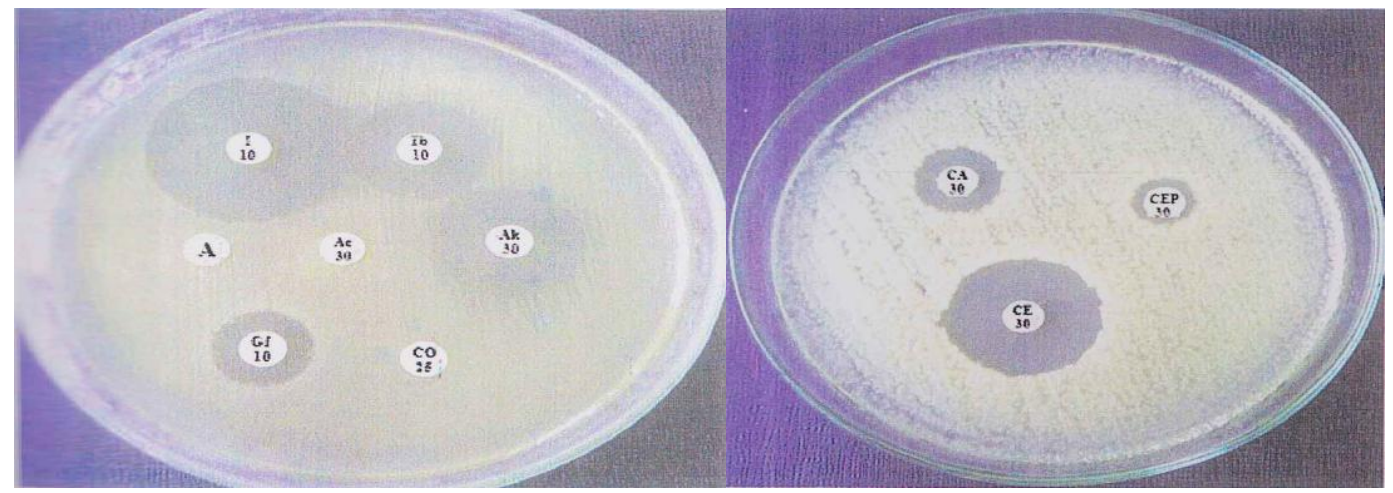

[ A- 6mm (R), Ac - 6mm (R), Ak-21mm(S), I - 30mm(S), Cf- 13mm (R), Nt- 20mm (S),Co - 6mm(R), CA $14 \mathrm{~mm}(\mathrm{R}), \mathrm{CE}-21 \mathrm{~mm}(\mathrm{R}), \mathrm{CEP}-8 \mathrm{~mm}(\mathrm{R})]$

Fig.2 Test strain positive on Double Disk Diffusion Synergy Test (DDST)

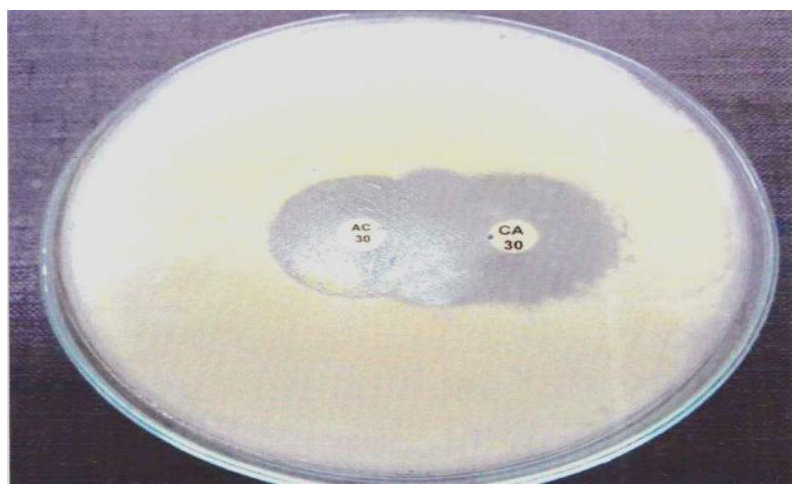


Graph.1 Percentage distribution of Enterobacteriaceae isolated from various pus samples

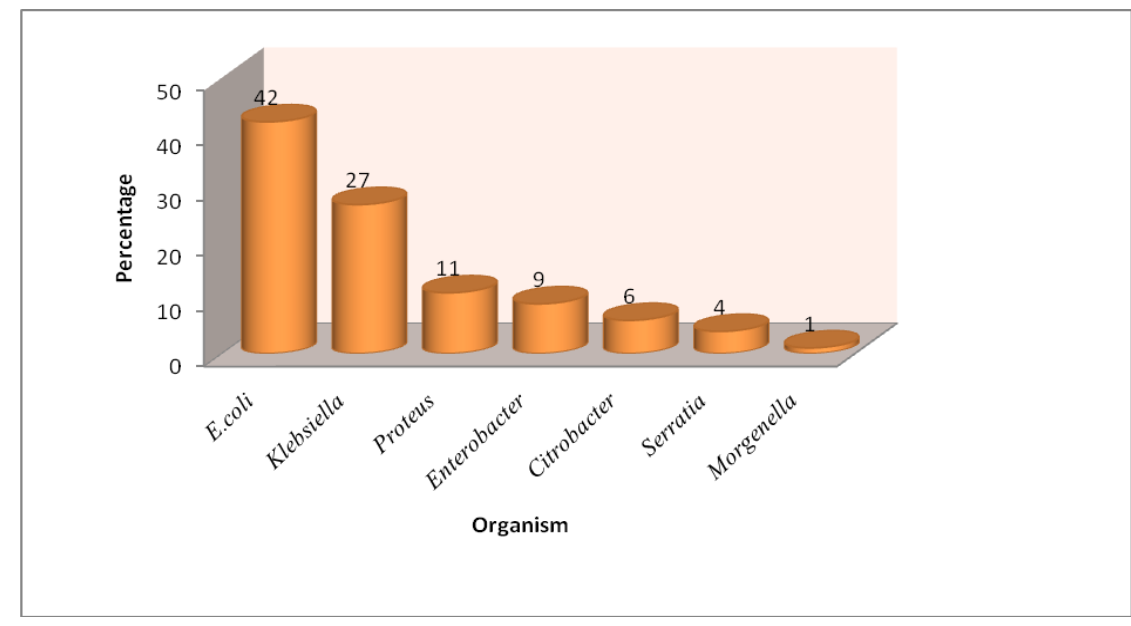

Graph.2 Percentage of ESBL producers among Enterobacteriaceae

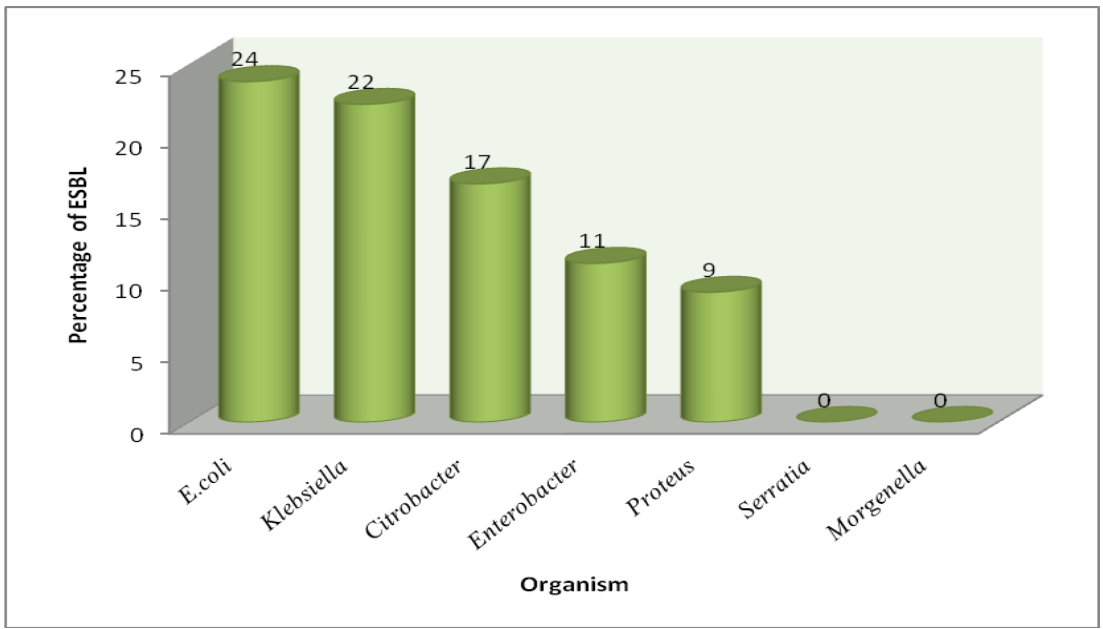

Graph.3 ESBL producers among various clinical conditions

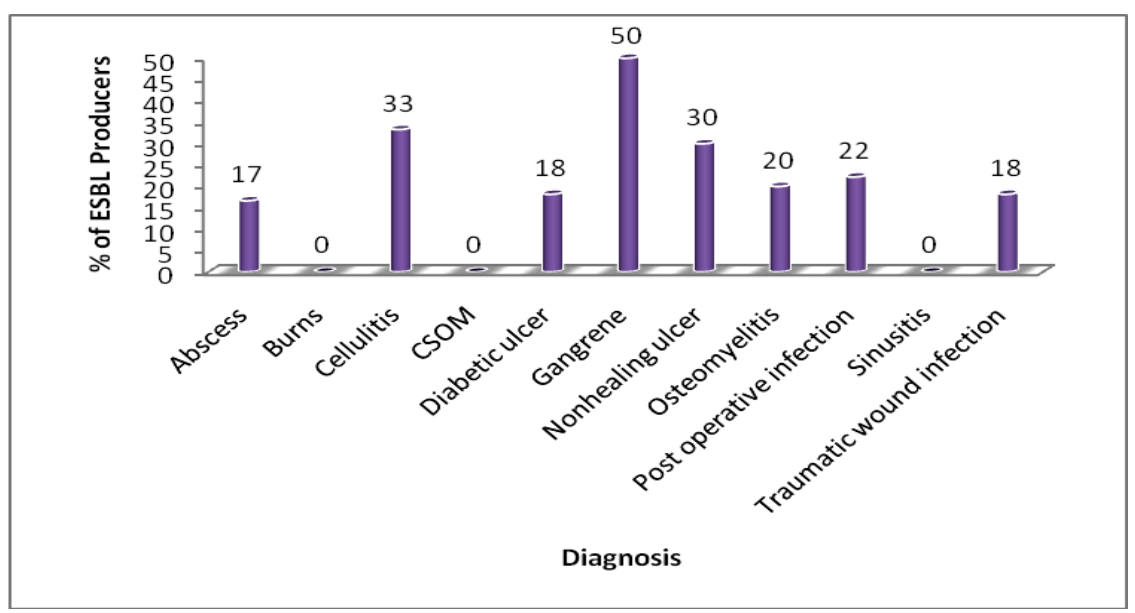


Graph.4 Antibiotic sensitivity and resistance pattern of ESBL producers

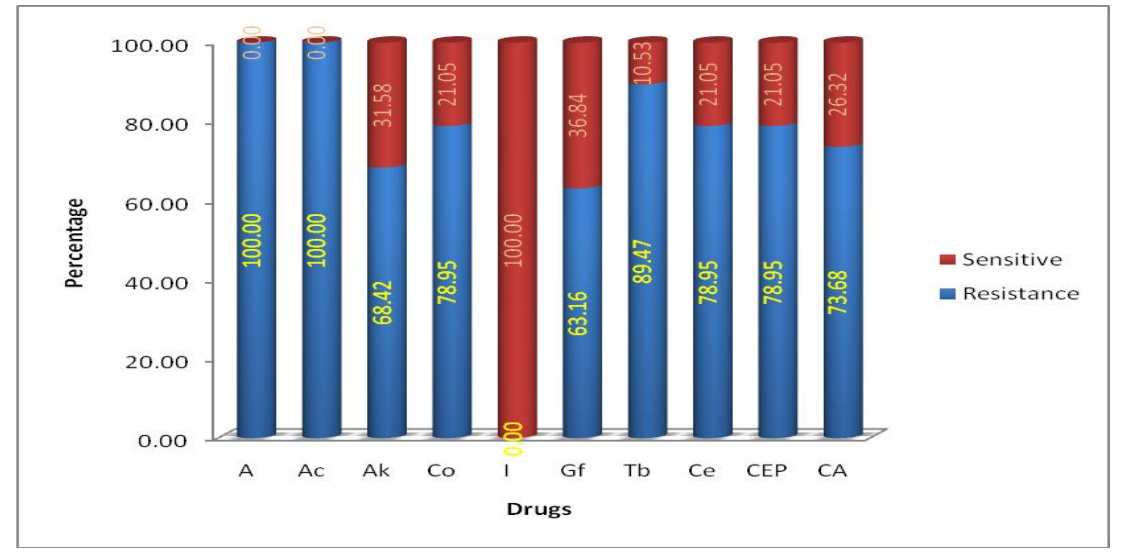

The discovery and development of antibiotics was undoubtedly one of the greatest advances of modern medicine. Unfortunately the emergence of antibiotic resistance bacteria is threatening the effectiveness of many antimicrobials. This has increased the hospital stay of the patients which in turn causes economic burden.

Patients with pyogenic infections admitted or attending out-patient department between November 2009 and November 2010 were included in this study.

Pus samples were collected from 100 patients with suspected pyogenic infections. The male to female ratio was $1.32: 1$. A similar observation was made by Ashwin $\mathrm{N}$ Ananthakrishnan et al., ${ }^{5}$ who have reported a male to female ratio of 1.78:1.

E.coli (42\%) was the commonest organism isolated, followed by Klebsiella (27\%), and Proteus species $(11 \%)$. Similar results were shown by study conducted by Bomasang et al., ${ }^{6}$ (45.8\% of E. coli) and Ananthakrishnan et al., ${ }^{5}$ (21\% of E. coli). Chan et al., reported $61 \%$ E. coli and $16 \%$ Klebsiella spp. ${ }^{7}$

However, in contrast to our study, in a study by Acharya et al., Klebsiella were the major pathogens, but the study group comprised of children which probably was the reason for the difference.

In the present study, Majority of ESBL producer showed resistance to Ampicillin (100\%), and Amoxyclav (100\%) followed by resistance to cotrimoxazole (78.95\%). Resistance to Amikacin was 68\%. Similar resistance rates were shown in the studies by Babypadmini et al., ${ }^{9}$, Menon et al., ${ }^{10}$ and Emily et al., ${ }^{6}$.

In our study, all the ESBL strains were sensitive to Imipenem(100\%). Similar results were observed by Babypadmini et al., ${ }^{9}$, T Menon et al., ${ }^{10}$, Shubha et al., ${ }^{11}$, Vinod kumar et al., ${ }^{12}$.

In present study, 19 isolates were reported as ESBL producers and hence should be resistance to $3^{\text {rd }}$ generation cephalosporins, but $26.3 \%$ were susceptible to Ceftazidime. Similarly Khurana et al., ${ }^{13}$ and Vinod Kumar et al., ${ }^{12}$ reported approximately 30\% of ESBL producers showing false susceptibility to $3^{\text {rd }}$ generation Cephalosporins. Ananthakrishnan et al., ${ }^{5}$ reported $53 \%$ of ESBL producers sensitive to Cefotaxime. Babypadmini et al., ${ }^{9}$ reported $14 \%$ and $12 \%$ of ESBL strains showing false susceptibility to Ceftazidime and Cefotaxime. All the above findings suggest the importance of detecting 
ESBLs in the laboratory, which helps in proper selection of antibiotics.

ESBL producers showed wide resistance to the non $\beta$-lactam antibiotics. In the present study $68.4 \%$ showed resistance to Amikacin and $63 \%$ to Gatifloxacin. Ananthankrishan et al., 5 showed $81.25 \%$ resistance to Gentamycin and $90.62 \%$ resistance to ciprofloxacin. Above findings can be comparable to the present study.

In the present study, all the ESBL producers were isolated from in-patients only. This indicates the prevalence of ESBLs among the bacteria of family Enterobacteriaceae was higher in isolates obtained from nosocomial patients as compared to outpatient isolates. Similarly study by Aamer Ali Shah et al., ${ }^{14}$ reported $37.5 \%$ of ESBLs in nosocomial and $6 \%$ in outpatient isolates.

In the present study, most important risk factor was instrumentation or surgery like amputation, wound debridement, drainage of abscess, laparotomy. Second important risk factor was long term hospital stay with exposure to $3^{\text {rd }}$ generation Cephalosporins. The information regarding ESBL producers, their susceptibility pattern, and risk factors were informed to the consultants who help them to select the proper antibiotics.

Multidrug resistance in ESBLs is a common problem in hospitals as seen in our study also, which emphasizes the need for judicious use of antimicrobial agents and their continuous in- vitro monitoring.

The routine susceptibility test done by clinical laboratories fail to detect ESBL positive strains and can erroneously detect isolates sometimes to be sensitive to any of the third generation cephalosporins, hence a special phenotypic confirmatory test (DDST) is indispensable for detecting ESBLs.
In conclusion differences in susceptibility pattern of organisms and frequency of infections between hospitals and communities make knowledge of local prevalence and resistance data extremely important.

With the spread of ESBL producing strains in hospitals all over the world, it is necessary to know their prevalence in a hospital so as to formulate antibiotic policy of empirical therapy in high risk units. There is a need for all diagnostic laboratories to perform ESBL detection as routine practice among all the Enterobacteriaceae members.

To overcome the problem of emerging ESBLs, combined interaction and cooperation of microbiologists, clinicians and infection control team are needed.

\section{References}

1. Chaudhary U, Aggarwal R. Extended spectrum $\beta$-lactamases (ESBL) - An emerging threat to clinical therapeutics. Indian J Med Microbiol 2004; 22(2): 7580.

2. Paterson DL, Bonomo RA. Extended spectrum $\beta$-lactamases: a clinical update. Clin Microbiol Rev 2005; 18(4): 657686.

3. Rodrigues C, Joshi P, Jani SH, Alponse M, Radhakrishnan R, Mehta A. Detection of $\beta$-lactamases in nosocomial gram negative clinical isolates. Indian journal of medical microbial 2004; 22(4): 247250.

4. Duttaroy B, Mehta S. Extended spectrum $\beta$-lactamases (ESBL) in clinical isolates of Klebsiella pneumoniae and Escherichia coli. Indian J Pathol Microbiol 2005; 48(1): 45 - 48.

5. Ananthakrishnan Ashwin N, Kanugo R, Kumar A, Badrinath S. Detection of extended spectrum $\beta$-lactamase producers among surgical wound infections and 
burns patients in JIPMER. Indian J Med Microbiol 2000; 18(4): 160 - 135.

6. Bomasang Emily S, Mendoza Myrna T. Prevalence and risk factors associated with extended spectrum $\beta$-lactamase (ESBL) production among selected Enterobacteriaceae isolates at Philippine general hospital. Phil. J Microbiol infects Dis 2003; 32(4): $151-158$.

7. Chan DTM, Chan MK. Urinary tract infection in a female medical ward. Journal of Hong Kong Medical Association 1987; 39(4).

8. Acharya VN, Jadav SK. Urinary tract infection: current status. J Postgrad Med 1980; 26:95-98.

9. Padmini Baby S, Appalaraju B. Extended Spectrum $\beta$-lactamase in urinary isolates of E.coli and K.pneumoniae - Prevalence and susceptibility pattern in a tertiary care hospital. Indian Journal of Medical Microbiology 2004; 22 (3): 172-174.

10. Menon T, Bindu D, Kumar CPG, Nalini $S$, Thirunarayan MA. Comparison of double disk and three dimensional methods to screen for ESBL producers in a tertiary care hospital. Indian $\mathrm{J}$ Med Microbiol 2006; 24(2): 117-120.

11. Shubha A, Ananthan S. Extended Spectrum $\quad \beta$-lactamase mediated resistance to third generation cephalosporins among Klebsiella pneumoniae in Chennai. Indian Journal of Medical Microbiology 2002; 20(2): 92 -95 .

12. Vinod Kumar CS, Neelagund YF. Extended $\quad$ Spectrum $\quad \beta$-lactamase mediated resistance to $3^{\text {rd }}$ generation cephalosporins among Klebsiella pneumoniae in neonatal septicaemia. Indian Paediatrics 2004; (41): 97 - 99.

13. Khurana S, Taneja N, Sharma M. Extended spectrum $\beta$-lactamase mediated resistance in urinary isolates of family Enterobacteriaceae. Indian $\mathbf{J}$ Med Res 2002; 116: 145 - 149.

14. Amer Ali Shah, Fariha Hasan. Prevalence of extended spectrum $\beta$ lactamase in nosocomial and outpatients (Ambulatory).

Pak.J.Med.Sci.2003; (193): $187-191$.

\section{How to cite this article:}

Shobha Medegar, K.R., Y.K. Harshika and Asha B. Patil. 2019. Detection of Extended Spectrum Betalactamases (ESBLs) Producing Enterobacteriaceae from Clinical Samples of Pus. Int.J.Curr.Microbiol.App.Sci. 8(01): 1369-1376.

doi: https://doi.org/10.20546/ijcmas.2019.801.145 